> Les enfants atteints de maladies neuromusculaires nécessitent parfois une assistance ventilatoire au long cours. Parmi les techniques employées, la ventilation sur trachéotomie reste par définition invasive et à même de mettre en péril l'intégration scolaire du jeune malade. <

Les maladies neuromusculaires sont responsables d'une faiblesse musculaire pouvant nécessiter, le cas échéant, une assistance ventilatoire au long cours [1-5]. Beaucoup d'entre elles concernent des enfants d'âge scolaire. Quand elle s'avère nécessaire, la ventilation dite non invasive (VNI) est généralement la technique proposée en première intention [6]. La VNI peut toutefois s'avérer insuffisante, notamment en cas d'évolution péjorative de la maladie, et un recours à la ventilation invasive sur trachéotomie est alors à envisager $[3,6-10]$.

Les enfants atteints de maladie neuromusculaire ont le plus souvent des fonctions cognitives préservées. Le maintien d'une scolarité est donc primordial pour eux. Leur scolarisation en milieu scolaire «classique », aussi dit ordinaire, est à privilégier: classes «ordinaires », ou classes spécialisées comme les ULIS (Unité Localisée pour l'Insertion Scolaire) ou les SEGPA (Section d'Enseignement Général et Professionnel Adapté). La trachéotomie reste un geste par définition invasif et elle peut peser/impacter significativement sur la vie scolaire de l'enfant. Les élèves ayant subi une trachéotomie doivent être accompagnés durant les temps scolaires et périscolaires par une personne ayant été formée aux aspirations endotrachéales (décret n 99-426 du 27 mai 1999). Dans le cadre de l'école, les accompagnants sont des AESH (Accompagnant d'Élèves en Situation de Handicap). Peu d'études ont évalué le retentissement de la ventilation invasive sur la scolarisation de l'enfant et les besoins spécifiques pour les aides mises en place durant les temps scolaires et périscolaires.

L'objectif principal de ce travail était de présenter un état des lieux de la scolarisation en milieu ordinaire des

\section{Trachéotomie chez les enfants atteints de maladies neuromusculaires et scolarisation en milieu ordinaire}

\section{Sont-elles compatibles?}

\author{
Laure Bonraisin ${ }^{1}$, Cécile Destremaut ${ }^{1}$, \\ Emmanuelle Lagrue ${ }^{1-3}$
}

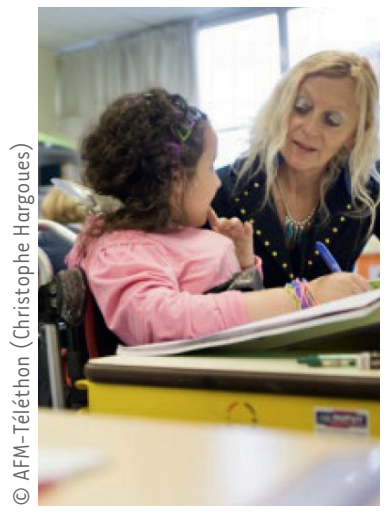

${ }^{1}$ CHRU Tours, Neuropédiatrie et Handicaps, Tours, France. ${ }^{2}$ Université François Rabelais de Tours, France.

${ }^{3}$ Inserm U1253, Tours, France. Emmanuelle.lagrue@inserm.fr

enfants trachéotomisés pour cause d'insuffisance respiratoire d'origine neuromusculaire. L'objectif secondaire était, face aux échecs de scolarisation rencontrés, d'en comprendre les raisons, afin de tenter de déterminer si ces échecs étaient bien en lien avec la trachéotomie elle-même.

\section{Matériel et méthodes}

Une étude épidémiologique observationnelle, descriptive et multicentrique, a été réalisée en France entre septembre 2018 et février 2019. L'enfant était inclus dans l'étude s'il avait dû être trachéotomisé dans un contexte d'insuffisance respiratoire de cause neuromusculaire et s'il était ou avait été scolarisé en milieu scolaire « classique » (classes ordinaires, ULIS, SEGPA). Afin de confronter les points de vue de personnes du milieu médical (Centres de compétence et de référence neuromusculaires $[C R]$ ), et associatif (Services régionaux de l'AFMTéléthon [SR-AFM]), ainsi que de l'éducation Nationale (médecins conseillers techniques des académies) [CTA], nous avons réalisé deux questionnaires: I'un était destiné aux CTA, l'autre aux CR et SR-AFM. Celui destiné aux CTA portait sur la formation des accompagnants, les 
gestes médicaux autorisés, les personnes susceptibles de pallier l'absence éventuelle d'AESH, le vécu des accompagnants de ces enfants, les modalités de suivi de la scolarisation et les raisons éventuelles de son échec. Le questionnaire destiné aux CR et SR-AFM portait sur le nombre d'enfants porteurs d'une trachéotomie pour cause de maladie neuromusculaire, les raisons du choix de la trachéotomie, le nombre d'enfants trachéotomisés et scolarisés en milieu scolaire «classique », le nombre d'enfants trachéotomisés ayant une A\&SH et les solutions pour pallier son absence, le recencement des échecs de scolarisation, leurs raisons et les solutions alternatives éventuellement mises en place, et enfin l'avis de ces professionnels concernant la trachéotomie comme éventuel frein à la scolarisation de ces enfants.

\section{Résultats}

\section{Questionnaire destiné aux CTA}

Sur les 30 médecins-conseillers techniques des académies contactés sur l'ensemble du territoire français, 14 (46,7\%) ont répondu. Le nombre d'enfants trachéotomisés (toutes pathologies neuromusculaires confondues) et scolarisés variait de 0 à 5 par académie soient 16 enfants au total pour l'ensemble des 14 académies ayant répondu.

Des différences notables au niveau inter et intra-régional ont été relevées dans la formation des AESH. Dans certaines académies, la formation était assurée par des Instituts de Formation en Soins Infirmiers. Dans d'autres, elle était assurée par la Croix-Rouge. Pour certaines, les AESH faisaient un stage dans un service de soins, associé ou non à une formation théorique complémentaire. L'académie de Lyon faisait appel à une association de soins à domicile et d'assistance médicotechnique. Dans les académies de Montpellier et de ClermontFerrand, les AESH n'avaient pas reçu de formation spécifique. Sur le plan intra régional, on observait que dans l'académie de Rennes, une formation spécifique était prévue dans un département et inexistante dans l'autre.

Gestes réalisés par l'AESH. Dans les académies où elles avaient reçu une formation spécifique, les A\&SH étaient autorisées à réaliser des aspirations endotrachéales, à l'exception des Côtes-d'Armor (alors même que ce geste était autorisé dans le Finistère, au sein de la même académie). L'académie de Clermont-Ferrand n'autorisait pas ses AESH à pratiquer ces gestes car elles n'avaient pas reçu de formation appropriée. Le choix opposé avait été fait par l'académie de Montpellier (gestes autorisés malgré l'absence de formation).

Allocation d'AESH. Parmi les académies ayant déclaré accueillir au moins un enfant trachéotomisé atteint de maladie neuromusculaire, les académies d'Amiens, Clermont-Ferrand et Lyon avaient fait le choix de la notification systématique d'une AESH, à la différence des académies de Dijon, Montpellier, Orléans-Tours et Rennes où cela n'était pas systématique.

Ressenti des AESH. Les académies de Bordeaux, Dijon, Montpellier et Rennes évoquaient des retours d'expérience assez négatifs des AESH, à la différence des académies de Lyon et Paris pour lesquelles les AESH n'avaient pas d'inquiétude majeure quant à la prise en charge de ces enfants.
Suivi de ces enfants. Dans toutes les académies, il y avait un suivi de ces enfants par une Équipe de Suivi de Scolarisation (ESS) et ce de manière annuelle.

Raisons des échecs de scolarisation. Pour l'académie de Dijon, les échecs étaient dus à la fois à des causes logistiques (éloignement trop important entre le domicile de l'enfant et l'école) et médicales (lourdeur de prise en charge pour l'AESH, absence d'AESH liée à des problèmes de formation et à la précarité économique de certaines d'entre elles). Les raisons des échecs étaient exclusivement médicales pour les académies de Bordeaux, Montpellier, Orléans-Tours et Rennes.

\section{Questionnaire destiné aux CR/SR-AFM}

Sur 26 CR et 19 SR-AFM contactés, 33 (73,3\%) ont répondu. Cent-six enfants ont pu être recensés. Le nombre moyen d'enfants connus par CR ou SR-AFM était de $3,21 \pm 2,9$. Nous avons constitué 2 groupes: le groupe $A$ 《 1 enfant ayant une trachéotomie/département » et le groupe $B$ « $\leq 1$ enfant ayant une trachéotomie/département ».

Les résultats du questionnaire figurent dans le Tableau $I$. Dans le groupe $A$, les échecs de scolarisation étaient médicaux (soins trop lourds pour l'AESH) pour 6 CR/ SR-AFM (100\%) et logistiques (distance importante domicile-école) pour 4 CR/SR-AFM $(66,7 \%)$. Dans le groupe $B$, les échecs de scolarisation étaient médicaux pour 5 CR /SR-AFM $(62,5 \%)$ et logistiques pour $4(50 \%)$ CR/SR-AFM. Parmi ces quatre échecs logistiques dans le groupe $B$, trois étaient primitivement scolaires, par refus de l'Éducation Nationale de scolariser l'enfant (Figure I).

\section{Discussion}

Cette étude, réalisée à l'échelle nationale, avait pour but de réfléchir aux conséquences sur la scolarisation en milieu scolaire ordinaire de la pratique d'une trachéotomie en contexte d'insuffisance respiratoire secondaire à une MNM.

Certains éléments pointent vers le fait que la trachéotomie constitue un frein à la scolarisation de ces enfants. La présence d'une AESH pour accompagner ces enfants est pourtant un facteur majeur de réussite pour leur intégration à l'école. L'absence de mise en place systématique d'un accompagnant (pour les aspirations endotrachéales notamment) a donc des conséquences délétères importantes, avec un risque élevé d'échec scolaire. En comparaison avec d'autres enfants (ayant une AESH pour un trouble déficitaire de l'attention par exemple), ce risque est aggravé par la lourdeur du handicap moteur et par l'absence partielle d'autonomie. II 


\begin{tabular}{|c|c|c|c|c|}
\hline \multirow[b]{3}{*}{ Nombre d'enfants trachéotomisés suivis par les $C R / S R-A F M^{1}$} & \multirow{3}{*}{$\begin{array}{c}\text { TOTAL } \\
\begin{array}{c}\text { (N = } 31 \text { CR/ } \\
\text { SRAFM)† } \\
106\end{array}\end{array}$} & \multicolumn{3}{|c|}{$\begin{array}{c}\text { Selon le nombre d'enfants } \\
\text { par départements couverts } \\
\text { par le CR/SR-AFM }\end{array}$} \\
\hline & & $\begin{array}{l}\text { Groupe A } \\
(N=12)\end{array}$ & $\begin{array}{l}\text { Groupe B } \\
(N=19) \dagger\end{array}$ & $\mathbf{P}$ \\
\hline & & 60 & 46 & - \\
\hline \multicolumn{5}{|l|}{ Nombre d'enfants $(\mathrm{N}, \%)$ : } \\
\hline Scolarisés en milieu scolaire ordinaire & $61(58,1 \%)^{2}$ & $34(56,7 \%)$ & $27(60,0 \%)^{3}$ & 0,73 \\
\hline Dans un niveau adapté & $55(52,4 \%)^{2}$ & $31(51,7 \%)$ & $24(53,3 \%)^{3}$ & 0,87 \\
\hline Avec présence d'une A\&SH & $53(50,5 \%)^{2}$ & $27(45,0 \%)$ & $26(57,8 \%)^{3}$ & 0,19 \\
\hline \multicolumn{5}{|l|}{ Personne suppléant l'AESH en son absence selon les CR/SR-AFM $(\mathrm{N}, \%)$ * } \\
\hline Parent & $7(22,6 \%)$ & $4(33,3 \%)$ & $3(15,8 \%)$ & \\
\hline Infirmier/éducateur & $3(9,7 \%)$ & $2(16,7 \%)$ & $1(5,3 \%)$ & 0,58 \\
\hline Aucune personne suppléante & $3(9,7 \%)$ & $1(8,3 \%)$ & $2(10,5 \%)$ & \\
\hline NR & $19(61,3 \%)$ & $6(50,0 \%)$ & $13(68,4 \%)$ & \\
\hline $\begin{array}{l}\text { Antécédents d'enfants en échec de scolarisation en } \\
\text { milieu ordinaire en lien avec la trachéotomie selon les } \\
\text { CR/SR-AFM }(\mathrm{N}, \%)\end{array}$ & $14(45,2 \%)$ & $6(50,0 \%)$ & $8(42,1 \%)$ & 0,76 \\
\hline Nombre d'enfants concernés ( $\mathrm{N}, \%)$ & $16(50,0 \%)^{4}$ & $4(33,3 \%)^{5}$ & $12(60,0 \%)^{6}$ & 0,27 \\
\hline \multicolumn{5}{|l|}{ Solution appliquée face à l'échec selon les } \\
\hline Scolarisation à domicile & $9(64,3 \%)$ & $5(83,3 \%)$ & $4(50,0 \%)$ & \\
\hline Scolarisation en IEM & $3(21,4 \%)$ & $1(16,7 \%)$ & $2(25,0 \%)$ & 0,83 \\
\hline Rééducation/SSR & $6(42,9 \%)$ & $2(33,3 \%)$ & $4(50,0 \%)$ & \\
\hline NR & $1(7,1 \%)$ & 0 & $1(12,5 \%)$ & \\
\hline NR & $1(3,2 \%)$ & 0 & $1(5,3 \%)$ & \\
\hline Trachéotomie constituant un frein à la scolarisation selon les CR/SR-AFM ( $\mathrm{N} \%)$ & $18(58,1 \%)$ & $5(41,7 \%)$ & $13(68,4 \%)$ & 0,14 \\
\hline
\end{tabular}

Tableau I. Caractéristiques de la population totale (CR/SR-AFM) et des groupes selon le nombre d'enfants trachéotomisés par département.

$N R=$ Non renseigné.

*Résultats non exclusifs, un même centre / AFM a pu donner plusieurs réponses (questionnaire à choix multiples).

$\dagger$ Exclusion de 2 centres sans suivi d'enfant trachéotomisé.

${ }^{1}$ Certains enfants peuvent avoir été comptabilisés 2 fois car suivis à la fois par le centre et par l'AFM de son lieu de résidence.

2Dénominateur «nombre d'enfants trachéotomisés » ramené à 105 ( 1 centre suivant 1 enfant n’ayant pas renseigné la question).

${ }^{3}$ Dénominateur «nombre d'enfants trachéotomisés » ramené à 45 ( 1 centre suivant 1 enfant n'ayant pas renseigné la question).

${ }^{4}$ Dénominateur «nombre d'enfants suivis par les centres ayant connu des échecs de scolarisation » de 55 ramené à 32 ( 3 centres ayant rencontré des échecs de scolarisation en milieu classique en lien avec la trachéotomie et suivant 23 enfants n'ayant pas renseigné la question).

${ }^{5}$ Dénominateur «nombre d'enfants suivis par les centres ayant connu des échecs de scolarisation » de 35 ramené à 12 (3 centres ayant rencontré des échecs de scolarisation en milieu classique en lien avec la trachéotomie et suivant 23 enfants n'ayant pas renseigné la question).

${ }^{6}$ Dénominateur «nombre d'enfants suivis par les centres ayant connus des échecs de scolarisation » de 20.

importe donc que l'augmentation des postes d'AESH soit une priorité de santé publique.

Cette enquête met également en évidence d'importantes disparités dans les pratiques selon les académies et les régions, et ce malgré une législation qui s'impose à tous.
Notre étude a toutefois des limites. Les résultats n'ont pas été statistiquement significatifs. II s'agit d'un travail portant sur une population de relative petite taille: un échantillon plus grand aurait possiblement permis d'améliorer la puissance de l'étude. 


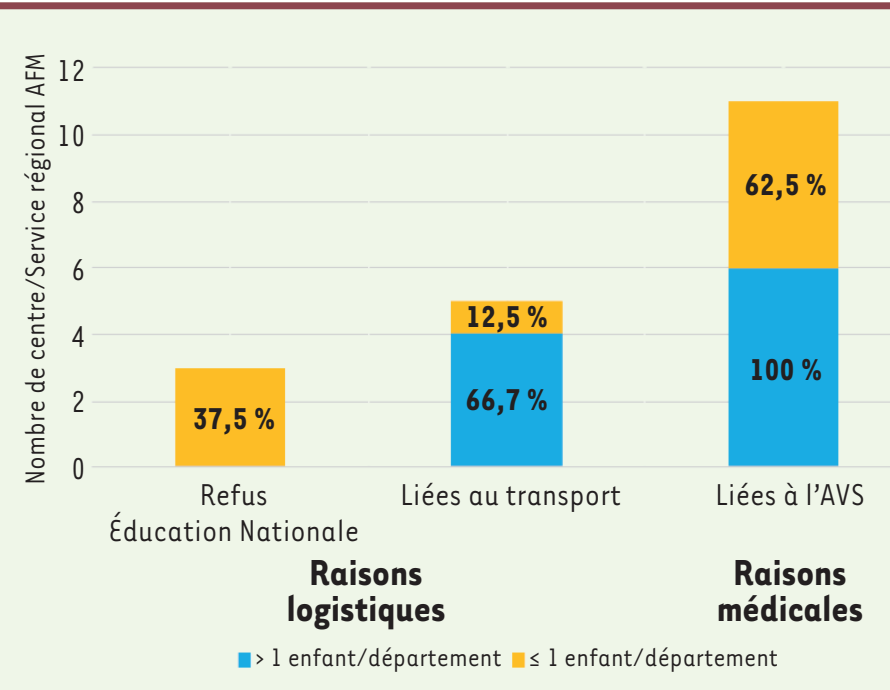

Figure 1. Causes d'échec de scolarisation en milieu classique liées à la trachéotomie (réponses par CR/SR-AFM).

Enfin, puisque la ventilation invasive sur trachéotomie semble bien avoir un impact négatif sur la scolarisation de ces enfants, des alternatives, comme par exemple la VNI avec utilisation d'un embout buccal (pipette) mériteraient d'être étudiées. Pour certains enfants ou adolescents, elles permettraient de surseoir à la trachéotomie et à ses conséquences collatérales négatives $\diamond$

\section{SUMMARY}

Tracheotomy in children with neuromuscular diseases and regular schooling: are they compatible?

Ventilating a young patient via a tracheostomy remains an invasive method to tackle the respiratory compromise often observed in several neuromuscular disorders. This approach significantly impacts the schooling of these children. The authors have surveyed professionals dealing with education, care, or social support nationwide. Regional discrepancies in practices of schooling in such situations are noted. Tracheostomy seems a major factor of exclusion out of the ordinary schooling system. $\diamond$

\section{LIENS D’INTÉRÊT}

Les auteurs déclarent n'avoir aucun lien d'intérêt concernant les données publiées dans cet article.

\section{GLOSSAIRE}

AESH : accompagnant d'élèves en situation de handicap

CR/SR-AFM : Centre de référence / Service régional AFM

CTA : conseiller technique des académies

ESS : équipe de suivi de scolarisation

SEGPA : section d'enseignement général et professionnel adapté

SSR : soins de suite et de réadaptation

ULIS : unité localisée pour l'insertion scolaire

\section{RÉFÉRENCES}

1. Boussaid G. Impact of respiratory care in patients with neuromuscular disease. Paris-Saclay : Thèse, 2016.

2. Han YJ, Park JD, Lee B, et al. Home mechanical ventilation childhood-onset hereditary neuromuscular diseases 13 years' experience at a single senter in Korea. PLoS One $2015 ; 10: 1-14$

3. Muir JF, Lamia B, Molano C, et al. Place de la trachéotomie au long cours dans l'insuffisance respiratoire chronique sévère à l'ère de la ventilation non invasive. Rev Mal Respir 2012 ; 29 : 994-1006.

4. Leger $P$, Paulus J. Modalités pratiques de la ventilation non invasive en pression positive, au long cours, à domicile, dans les maladies neuromusculaires, recommandations pour la pratique clinique. Rev Mal Respir 2006 ; $23: 1457-9$.

5. Orlikowski D, Prigent $\mathrm{H}$, Gonzales J, et al. Ventilation mécanique à domicile et au long cours des patients neuromusculaires (indication, mise en place et surveillance). Rev Mal Respir $2005 ; 22$ : 1021-30.

6. HAS et AFM. Modalités pratiques de la ventilation non invasive en pression positive, au long cours, à domicile, dans les maladies neuromusculaires. Recommandations de bonnes pratiques. HAS/AFM, 2006. www.has-sante.fr.

7. Birnkrant DJ, Bushby K, Bann CM, et al. Diagnosis and management of Duchenne muscular dystrophy, part 2: respiratory, cardiac, bone health, and orthopaedic management. Lancet Neurol 2018 ; 17 : 347-61.

8. Finkel RS, Mercuri $\varepsilon$, Meyer $\mathrm{OH}$, et al. Diagnosis and management of spinal muscular atrophy. Part 2: Pulmonary and acute care, medications, supplements and immunizations, other organ systems and ethics. Neuromuscul Disord $2018 ; 28$ : 197-207.

9. Priou P, Trzepizur W, Meslier N, Gagnadoux F. Mise au point dans la prise en charge respiratoire des maladies neuromusculaires chroniques. Rev Pneumol Clin 2017; $73: 316-22$

10. AFM-Téléthon. Trachéotomie et maladies neuromusculaires. Savoir et comprendre. AFM, 2007. www.afm-telethon.fr.

\section{TIRÉS À PART}

દ. Lagrue

\section{Retrouvez toutes les Actualités de la Myologie sur les sites de :}

la Société Française de Myologie

www.sfmyologie.org la filière de santé neuromusculaire FILNEMUS

www.filnemus.fr 